\title{
Hipertiroidismo en gestación. Clínica, morbi-mortalidad materna, fetal y perinatal.
}

\author{
Hiperthyroidism in pregnancy. Clinic characteristics and maternal, fetal and perinatal \\ morbility and mortality.
}

\author{
Palacios Porras, Hermógenes*, Solis Villanueva, José** \\ *Médico Cirujano. \\ **Profesor Asociado del Departamento de Medicina. Universidad Peruana Cayetano Heredia. \\ Jefe del Servicio de Endocrinología, Hospital Nacional Arzobispo Loayza. Lima, Perú.
}

\section{RESUMEN}

Objetivo: Determinar la prevalencia y características clínicas del hipertiroidismo en gestantes y definir la influencia de hipertiroidismo sobre la morbimortalidad materna, fetal y perinatal. Material y métodos: Se incluyeron 29 pacientes con diagnóstico de hipertiroidismo y gestación, que cumplieron los criterios de inclusión. Las pacientes fueron divididas en dos grupos, hipertiroidismo compensado durante toda o la mayor parte de la gestación (Grupo I, $\mathrm{n}=11$ ) y con hipertiroidismo no compensado (Grupo II, $n=18$ ). Resultados: La prevalencia de hipertiroidismo y gestación fue de $0.03 \%$. La enfermedad de Graves estuvo presente en el $96.5 \%$ de los casos; el $62 \%$ cursó con náuseas y vómitos y el $90 \%$ con bocio de 40 a 60 gramos. Comparando los grupos I y II, observamos en este último, una mayor frecuencia cardíaca y una menor ganancia de peso $(\mathrm{p}<0.001$ y $\mathrm{p}<0.0002$, respectivamente). Los productos del grupo II, tuvieron un mayor índice de prematuridad ( 5 de 7$)$ y de bajo peso al nacer $(\mathrm{p}<0.0003)$; todos los abortos (4), óbitos (2) y malformaciones congénitas (2), se presentaron en este grupo. El tratamiento recibido fue metimazol a dosis variable (10 a 30 $\mathrm{mg} /$ día), no encontramos efectos deletéreos importantes atribuibles al tratamiento. Conclusiones: El hipertiroidismo mal controlado durante la gestación produce mayor morbimortalidad materna, fetal y perinatal. (Rev Med Hered 1995; 6: 107-114).

PALABRAS CLAVE: Hipertiroidismo, gestación, síntomas, signos, tratamiento.

\section{SUMMARY}

Objective: To find the prevalence, clinic characteristics of hyperthyroidism during pregnancy 
and is influence upon maternal, fetal and perinatal morbility and mortality. Material and methods: We included 29 patients with a diagnosis of hyperthyroidism and pregnancy fulfilling the inclusion criteria. The patients were divided in tow groups. Compensated hyperthyroidism during all or most part of pregnancy (Group I, n=11) and not compensated hyperthyroidism (Group II, $\mathrm{n}=18$ ). Results: The prevalence of hyperthyroidism and pregnancy was $0.03 \%$. Graves disease was present in $95.5 \%$ of cases; $62 \%$ presented nausea and vomits and $90 \%$ showed diffuse goiter of 40-60 gr. of weight. Comparing groups I and II, we observed that group II mothers had on the average, a higher heart frequency and smaller gain of weight $(\mathrm{p}<0.001$ and $\mathrm{p}<0.002$ respectively). The offspring of group II had the highest prematurity rate (5 of 7) and low body weight ( $p<0.003)$; all abortions (4), demised (2) and congenit malformations (2) occurred in this group. The treatment employed was metamizol in variable schedule (10-30 mg/day). We did not find any important adverse effect of treatment. Conclusions: No compensated hyperthyroidism during pregnancy produced greatest maternal, fetal and perinatal mobility and mortality. (Rev Med Hered 1995; 6: 107-114).

KEY WORDS: Hyperthyroidism, pregnancy, symptoms, signs, treatment.

\section{INTRODUCCION}

El hipertiroidismo, constituye la patología tiroidea más frecuente de la gestación (1) y se presenta aproximadamente en el 0.05 al $0.2 \%$ de los embarazos $(2,3,4)$.

La causa más común de hipertiroidismo durante la gestación es la enfermedad de Graves $(6,7)$, otras causas incluyen al bocio nodular tóxico, tiroiditis crónica, enfermedad trofoblástica e hiperemesis gravídica $(3,7,8,9)$.

El diagnóstico del hipertiroidismo en la gestación tiene sus particularidades, ambas entidades comparten síntomas y signos comunes como: intolerancia al calor; nerviosismo, taquicardia y crecimiento de la glándula tiroidea; el hiperestrogenismo produce elevación de la globulina transportadora de hormonas tiroideas (TGB) y por consiguiente de los niveles circulantes de $\mathrm{T}_{4}$ y $\mathrm{T}_{3}(3,9,10,11)$, la confirmación diagnóstica requiere por ello de la medicación de las fracciones libres $\mathrm{T}_{4}$ y $\mathrm{T}_{3}$, del índice de tiroxina libre $\left(\mathrm{IT}_{4} \mathrm{~L}\right)$, o bien de $\mathrm{TSH}$ por ensayos inmunorradiométricos (IRMA); técnicas que con frecuencia no están disponibles en los hospitales de nuestro país, pero es posible obtener una confirmación diagnóstica aceptable cuando los valores de $\mathrm{T}_{3}$ y $\mathrm{T}_{4}$ totales son referidos a los valores normales de estas hormonas en gestantes. No controlar el hipertiroidismo en la gestación puede conducir la crisis tirotóxica, aborto, óbito fetal, prematuridad, bajo peso al nacer, hipertiroidismo fetal $\mathrm{y} / \mathrm{o}$ neonatal y malformaciones congénitas $(3,5,6)$.

Las series reportadas en este sentido son escasas, el número de pacientes con que cuentan es reducido y el tratamiento del hipertiroidismo presenta puntos de controversia que son necesarios definir.

En el presente trabajo, reportamos la experiencia sobre hipertiroidismo y gestación de los hospitales nacionales Arzobispo Loayza y Cayetano Heredia, en un período de 12 años, con el propósito de determinar, la prevalencia y características clínicas del hipertiroidismo en gestantes $\mathrm{y}$, de definir la influencia del hipertiroidismo sobre la morbimortalidad materna, fetal y perinatal. 


\section{MATERIAL Y METODOS}

Se Revisaron los archivos de las Oficinas de Estadística y de los Servicios de Endocrinología de los hospitales nacionales Arzobispo Loayza (HNAL) y Cayetano Heredia (HNCH), así como las historias clínicas correspondientes a un período de 12 años. En dicho período se atendieron 89,700 partos en ambos hospitales: 43,500 $(\mathrm{HNCH})$ y 46,200 $(\mathrm{HNCH})$, de este universo se ubicaron 79 pacientes con diagnóstico de hipertiroidismo y gestación de las cuales se descartaron 50 por o cumplir con los criterios de inclusión, las 29 pacientes hipertiroideas restantes tuvieron una o más gestaciones, haciendo un total de 32 embarazos, los cuales hemos analizado en forma retrospectiva.

Los criterios de inclusión fueron:

a. Cuadro clínicos de hipertiroidismo

b. Niveles de hormonas tiroideas elevadas durante la gestación. $\mathrm{T}_{4}$ mayor de $15 \mu / \mathrm{dl} \mathrm{T}_{3}$ mayor de $270 \mathrm{ng} / \mathrm{dl}$.

c. y/o antecedentes de diagnóstico definido de hipertiroidismo (clínico y bioquímico) previo a la gestación.

En el período de tiempo en que se atendieron las gestantes motivo del presente estudio, no eran disponibles en nuestros hospitales técnicas para medir las fracciones libres de $\mathrm{T}_{4}$ y $\mathrm{T}_{3}$ es por ello que se midieron los niveles totales de dichas hormonas y para determinar los niveles séricos de referencia de $\mathrm{T}_{3}$ y $\mathrm{T}_{4}$ que se indican, hemos realizado estas determinaciones en un grupo control de 45 gestantes normales, tomadas del azar, que acudieron a su control al Servicio de Obstetricia del HNAL, 15 por cada trimestre, también se determinó TSH, para una mejor definición del cuadro de eutiroidismo, en cada una de estas gestantes se tomó una muestra de sangre, los sueros fueron congelados a $-20^{\circ} \mathrm{C}$, hasta el día de su proceso. Las determinaciones se hicieron en el Laboratorio de Endocrinología del HNAL, por Radioinmunoanálisis, usando el sistema de fase sólida mediante Kits comerciales (Diagnostic Products Corp. Los Angeles, CA). Todas las muestras fueron procesadas por duplicado en un mismo ensayo, el CV intra ensayo fué de $6 \%$.

Los valores hormonales de las pacientes hipertiroideas fueron mayores de $20 \mu \mathrm{g} / \mathrm{dl}$ para $\mathrm{T}_{4} \mathrm{y}$ de $327 \mathrm{ng} / \mathrm{dl}$ para $\mathrm{T}_{3}$ al momento del diagnóstico. También se analizaron las historias clínicas de los 32 neonatos de las 29 pacientes hipertiroideas.

Los 32 casos de hipertiroidismo y gestación estudiada fueron divididos en 2 grupos.

\section{Grupo I}

Pacientes que estuvieron compensadas de su cuadro de hipertiroidismo, durante toda o la mayor parte de la gestación y al momento del parto $n=11$ (37.9\%) (13 gestaciones). Se consideró compensada cuando clínicamente lucía entiroidea y tenía valores de $\mathrm{T}_{4} \mathrm{o} \mathrm{T}_{3}$ por debajo de $15 \mu \mathrm{g} / \mathrm{dl}$ y $270 \mathrm{hg} / \mathrm{dl}$ respectivamente por lo menos durante la segunda mitad del embarazo.

\section{Grupo II}

Pacientes que estuvieron hipertiroideas durante la gestación y al momento del parto $n=18$ $(62.1 \%)$ (19 productos, un embarazo fue general).

En los análisis estadísticos se utilizó la prueba de Chi cuadrado para variables discretas, 
corrección de Yates para valores menores de 5 y análisis de varianza para variables contínuas.

\section{RESULTADOS}

De los 89,700 partos atendidos en ambos hospitales (HNAL Y HNCH) en los 12 últimos años la prevalencia de hipertiroidismo y gestación que encontramos fué de $0.03 \%$ analizando separadamente cada hospital ésta fue de $0.04 \%$ en el HNAL (19 casos) y de $0.02 \%$ en el HNCH (10 casos).

La edad promedio de las gestantes hipertiroideas fué de 30.8 años (rango: 18 y 39 a), un poco mayor que la edad promedio del grupo de gestantes normales 26.3 (rango: 16 a 38 a). No hubo diferencia significativa entre las edades de los grupos I y II.

El tiempo de la enfermedad antes de la gestación fue de menos de un mes de 2 años, con un promedio de 11.2 meses. El diagnóstico de hipertiroidismo se estableció antes de la gestación en 17 de las 29 pacientes.

La causa del hipertiroidismo en el $96.5 \%$ de las gestantes estudiadas, fue la enfermedad de Graves y en el 3.5\% restante bocio nodular tóxico.

Los síntomas y signos de mayor frecuencia se describen en las tabla $\mathrm{N}^{\circ} 1$ y tabla $\mathrm{N}^{\circ} 2$; es de destacar que un elevado porcentaje de pacientes $(60 \%)$ presentan náuseas y vómitos y en un grupo significativo de ellas (12.5\%) éstos fueron severos y catalogados como hiperemesis gravídica; el bocio estuvo presente en el $90 \%$ de los pacientes y en el $88 \%$ delos casos su tamaño fue moderado con un peso entre 40 y 60 gr.

\begin{tabular}{|ll|}
\hline $\begin{array}{c}\text { Tabla N} \text { } 1 \text { 1. Hipertiroidismo y gestación } \\
\text { Síntomas de mayor frecuencia. }\end{array}$ \\
\hline & $\%$ \\
\hline Nerviosismo & 62 \\
Náuseas y vómitos & 60 \\
Palpitaciones & 38 \\
Disnea & 33 \\
Disminución de apetito & 31 \\
Caída de cabello & 25 \\
Diarrea & 18 \\
Labilidad emocional & 12 \\
Dolor abdominal & 12 \\
Otros & 10 \\
\hline
\end{tabular}

\begin{tabular}{|c|c|c|}
\hline \multicolumn{3}{|c|}{\begin{tabular}{|c|} 
Tabla N*2. Hipertiroidismo y gestación \\
Signos de mayor frecuencia.
\end{tabular}} \\
\hline BOCto & & 90.0 \\
\hline - Leve $(40 \mathrm{gr})$ & $30 \%$ & \\
\hline - Moderado $(60 \mathrm{gr})$ & $38 \%$ & \\
\hline - Severo $(80 \mathrm{gr})$ & $12 \%$ & \\
\hline TREMOR DISTAL. DE MIEMBROS & & 82.0 \\
\hline TAQUICARDIA & & 79.0 \\
\hline HIPERREFLEXIA & & 55.0 \\
\hline EXOFTALMOS & & 44.0 \\
\hline - Leve & $87 \%$ & \\
\hline - Moderado & $13 \%$ & \\
\hline SOPLO EN EL BOCIO & & 15.0 \\
\hline ARRITMIA CARDIACA & & 3.2 \\
\hline ONICOLISIS & & 5.0 \\
\hline otros & & 8.0 \\
\hline
\end{tabular}


Los valores promedio (x), séricos de $\mathrm{T}_{4}$ y $\mathrm{T}_{3}$ y TSH del grupo de gestantes normales $(\mathrm{n}=45)$ fueron $10.32 \mu \mathrm{g} / \mathrm{dl}(8.6-14) 190.24 \mathrm{ng} / \mathrm{dl}(160-270)$ y $2.61 \mathrm{UI} / \mathrm{ml}(1.7-6)$ respectivamente. Para determinar los límites superiores normales se usó $\mathrm{x}+2 \mathrm{DS}$ y éstos fueron: $15 \mu \mathrm{g} / \mathrm{dl}$ y 270 $\mathrm{ng} / \mathrm{dl}$, para $\mathrm{T}_{4} \mathrm{y} \mathrm{T}_{3}$ respectivamente, los niveles hormonales de las pacientes hipertiroideas al momento de su diangóstico por encima de $20.00 \mu \mathrm{g} / \mathrm{dl}$ para $\mathrm{T}_{4}$ y $327 \mathrm{ng} / \mathrm{dl}$ para $\mathrm{T}_{3}$; éstos valores son claramente superiores a los del grupo control. En el seguimientos de los niveles hormonales de los grupos I y II de gestantes hipertiroideas, éstos son mayores en el grupo II con diferencias estadísticamente significativas par $T_{3}$ en el primer trimestre $(p<0.037)$ y para $\mathrm{T}_{4}$ en el tercer trimestre $(\mathrm{p}<0.033)\left(\right.$ Tabla $\left.\mathrm{N}^{\circ} 3\right)$.

\begin{tabular}{|c|c|c|}
\hline & \multicolumn{2}{|c|}{ Tabla N*3. Nivel de hormonas tiroideas. } \\
\hline & GRUPOI & GRUPO॥ \\
\hline \multicolumn{3}{|l|}{$T 4$ ( $\mu \mathrm{g} / \mathrm{dl})$} \\
\hline Pre-Gestación & $22.31 \pm 4.86(6)$ & $23.80 \pm 5.67(10)$ \\
\hline 10 Trimestre & $20.50 \pm 6.44(6)$ & $28.25 \pm 11.14(4)$ \\
\hline 2 Trimestre & $19.85 \pm 7.01(5)$ & $22.00 \pm 2.80(2)$ \\
\hline 3. Trimestre & $13.33 \pm 1.52(3)$ & $22.85 \pm 3.76(7)^{\circ}$ \\
\hline \multicolumn{3}{|l|}{ T3 (ng/dl) } \\
\hline Pre-Gestación & $239.16 \pm 62.64(6)$ & $331.42 \pm 88.96(7)$ \\
\hline 1 Trimestre & $253.00 \pm 109.84(6)$ & $418.00 \pm 113.66(5) \&$ \\
\hline 2. Trimestre & $223.00 \pm 117.00(3)$ & $700.00 \pm 0.00$ (1) \\
\hline 3. Trimestre & & $326.00 \pm 124.62(8)$ \\
\hline 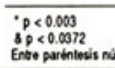 & prosionter. & \\
\hline
\end{tabular}

En la comparación de los grupos I y II, observamos que la frecuencia cardíaca fue mayor en las gestantes no compensadas de su hipertiroidismo (grupo II) con una $\mathrm{p}<0.035$ y una $\mathrm{p}<0.001$ para el segundo y tercer trimestre respectivamente y tres de ellas $(18.7 \%)$ desarrollaron insuficiencia cardíaca congestiva. La ganancia de peso durante la gestación fue claramente mayor en el grupo de pacientes compensadas de su hipertiroidismo (grupo I) con una $p<0.02$ y $p<0.03$ para el primer y segundo trimestre respectivamente (Figura $\mathrm{N}^{\circ} 1$ y figura $\mathrm{N}^{\mathrm{o}} 2$ ).

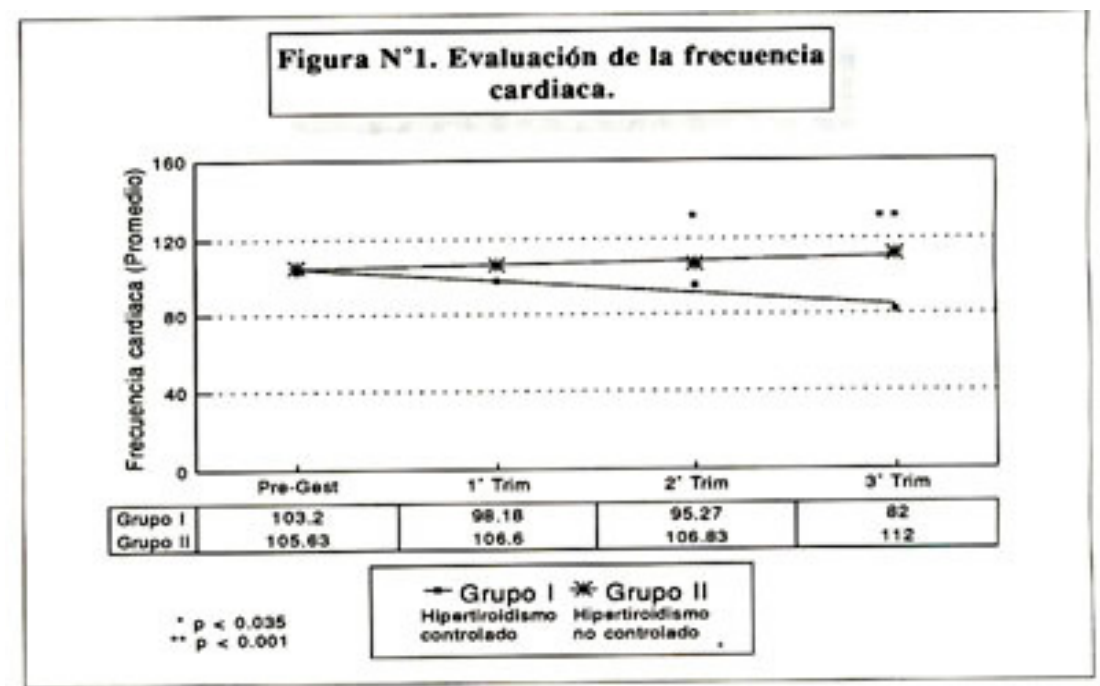




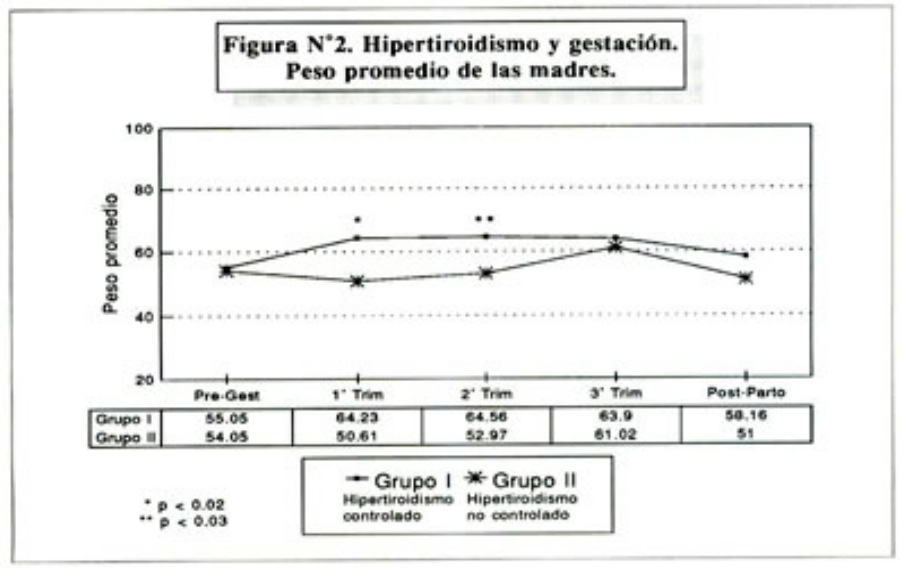

La condición de los productos fue diferente entre ambos grupos: la edad gestacional fue de $37.84 \pm 2.8$ semanas en el grupo I y $35.76 \pm 3.7$ en el grupo II; el peso promedio al nacer del grupo I fue de $2.69 \pm 0.67 \mathrm{Kg}$. Y $2.08 \pm 0.69 \mathrm{Kg}$. para el grupo II ( $<0.03)$, el $30.8 \%$ de los neonatos del grupo I fueron de bajo peso para su edad gestacional (BEG) y $46.6 \%$ de los neonatos del grupo II tuvieron esta condición; en el grupo I, de los neonatos, 2 (15.4\%) fueron prematuros y no se presentó ninguna otra complicación; en el grupo II de los 19 productos 5 fueron prematuros $(26.7 \%) 2$ natimuertos $(10.5 \%) 4$ abortos $(21.0 \%)$ y 2 tuvieron malformaciones congénitas severas ( $19 \%$ ) uno de ellos presentó microcefalia, gran cardiomegalia, micrognatia y abundante lanugo con un PN de 1650 gr, el otro mostró hidrocefalia meningocele lumbar moderado y malformaciones de miembros con PN de 3050 gr. ambos fallecieron poco tiempo después del nacimiento (Tabla $\mathrm{N}^{\circ} 4$ ).

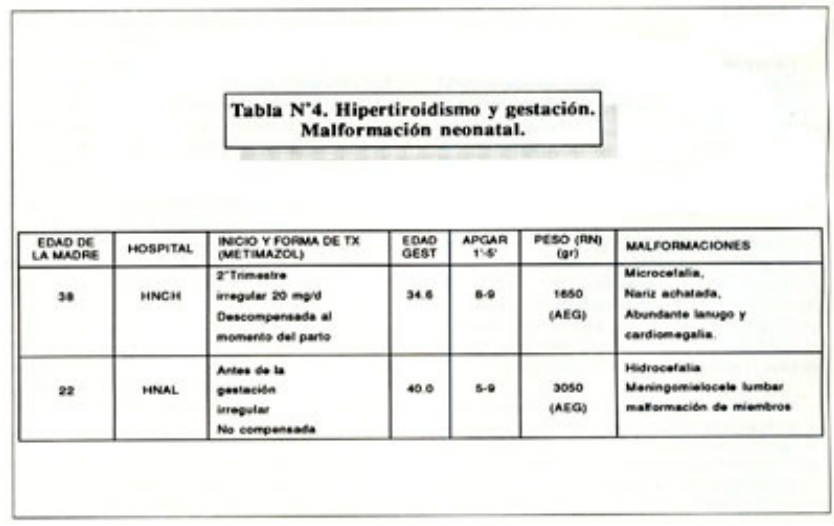

De los 26 neonatos que nacieron vivos, 2 presentaron bocio leve, ambos productos de un embarazo gemelar, la madre estuvo descompensada y recibió metimazol en forma regular a dosis de $20 \mathrm{mg} /$ día a partir del segundo trimestre, los productos fueron prematuros; un tercer producto, hizo hipotiroidismo primario transitorio confirmado por pruebas bioquímicas hormonales, la madre estuvo descompensada y recibió metimazol a dosis de $20 \mathrm{mg} / \mathrm{día}$ durante el tercer trimestre y yoduro (lugol) 20 gotas/día en el peri parto.

En lo relacionado al tratamiento, todas las pacientes del grupo I recibieron regularmente metimazol durante la gestación, a dosis variables en promedio de $21.9 \mathrm{mg} / \mathrm{día}$; un grupo 
significativo de los pacientes del grupo II recibió metimazol en forma no regular pero a dosis similar a la del grupo I. Un grupo de 9 gestantes de ambos grupos recibió propanolol durante el tercer trimestre a dosis promedio de $57.78 \mathrm{mg} /$ día. No encontramos efectos deletéreos atribuídos al tratamiento con ambas drogas ya sea sobre el curso de la gestación y/o el producto de la misma.

\section{DISCUSION}

La prevalencia de hipertiroidismo y gestación que hemos encontrado en el presente estudio $(0.03 \%)$ es menor que las reportadas en la literatura $(2,3,4)$ esto se debe posiblemente a que un grupo significativo de historias clínicas con el diagnóstico de hipertiroidismo y gestación fueron descartadas (50 de los 79) por no tener datos de laboratorio que confirmen el diangóstico.

Es conocido que en el hipertiroidismo existen alteraciones en el ciclo menstrual entre ellas la polimenorrea e hipomenorrea con ciclos anovulatorios, pero la fertilidad no está abolida, lo que hace necesario el uso de un método anticonceptivos eficaz en toda paciente hipertiroidea en edad reproductiva y vida sexual activa.

La causa más común de hipertiroidismo durante la gestación que hemos encontrado es la enfermedad de Graves y ocurrió en el $96.5 \%$, en la gran mayoría de los casos ésta estuvo presente antes de la gestación y el promedio de la enfermedad antes de iniciarse el embarazo fue de 11.2 meses. Estos hallazgos confirman lo reportado previamente $(3,7,8,10)$; en el $3.5 \%$ restante la causa fue el bocio nodular tóxico, su menor frecuencia se explica por su menor prevalencia en la población general y por que fundamentalmente ocurre en personas mayores de 40 años. En la literatura se han reportado otras causas como tiroiditis de Hashimoto, tiroiditis subaguda, mola hidatiforme e hiperemesis gravídica $(3,7,8,9,10)$ que nosotros no hemos evidenciado. Los síntomas y signos que presentaron los pacientes son los clásicos del hipertiroidismo del adulto joven pero es de destacarse la elevada prevalencia de náuseas y vómitos $(60 \%)$ y un grupo de ellas $(12.5 \%)$ fueron diagnosticadas de hiperemesis gravídica. En el diagnóstico diferencial debe tenerse en cuenta que ésta última generalmente no cursa con bocio ni exoftalmos, si bien es cierto que puede encontrarse hipertiroxinemia hasta en un $50 \%$ de los casos, pero en la mayoría de ellas no hay elevación de las fracciones libres (13) y en los casos de mayor dificultad diagnóstica los niveles de zinc eritrocitario normales en la hiperemesis gravídica y bajos en el hipertiroidismo pueden hacer el diangóstico (14); estos síntomas ceden cuando se resuelve el estado tirotóxico, y no se conoce la fisiopatología de las náuseas y vómitos en el hipertiroidismo asociado a la gestación.

Es conocido que durante la gestación por estímulo estrogénico hay un incremento de los niveles de TBC y por ende de los niveles totales de $\mathrm{T}_{3}$ y $\mathrm{T}_{4}(3,9,10)$ pero las fracciones libres de $\mathrm{T}_{3}\left(\mathrm{FT}_{3}\right)$ y $\mathrm{T}_{4}\left(\mathrm{FT}_{4}\right)$ no se alteran $(9,10)$ excepto durante las cinco primeras semanas de gestación, donde ocurre una leve elevación de las mismas, relacionada probablemente al efecto tiroestimulante de $\mathrm{HCG}(3,12)$. Este hecho hace necesario la determinación de $\mathrm{FT}_{4} \mathrm{y}$ $\mathrm{FT}_{3}$ o del índice del $\mathrm{T}_{4}$ libre $\left(\mathrm{IT}_{4} \mathrm{~L}\right)$ para definir el estado de función tiroidea, estas pruebas demandan más costos y no están disponibles en la mayoría de nuestros centros de atención médica, en cambio las determinaciones de $\mathrm{T}_{3}$ y $\mathrm{T}_{4}$ totales son de uso muchos más frecuente, por ello hicimos estas determinaciones en un grupo de 45 gestantes (15 por cada trimestre) con el objeto de tener valores referenciales de estas pruebas durante la gestación, considerando la x + 2DS, estos valores fueron: $270 \mathrm{ng} / \mathrm{dl}$ y $15 \mu \mathrm{g} / \mathrm{dl}$ para $\mathrm{T}_{3}$ y $\mathrm{T}_{4}$ respectivamente, ambos similares a los reportados anteriormente (8), valores por encima de 
éstos aunados a un cuadro clínico compatible hacer probable el diagnóstico de hipertiroidismo en la gestación, claro está si se cuenta con estudios más sensibles y específicos como lo mencionamos anteriormente éstos deben hacerse para establecer un diagnóstico definitivo de hipertiroidismo y gestación.

Al comparar los grupos (gestantes hipertiroideas compensadas) y grupo II (gestantes del grupo II, determinó en las madres una mayor frecuencia cardíaca y una menor ganancia de peso durante la gestación, e incluso tres de ellas hicieron insuficiencia cardíaca congestiva (18.7\%) respondiendo adecuadamente el tratamiento. En relación a los neonatos evidenciamos una menor edad gestacional, más bajo peso al nacer y una mayor prevalencia de prematuridad en el grupo II, así mismo todos los abortos y óbitos fetales ocurrieron en este grupo; estas diferencias fueron estadísticamente significativas lo cual nos indica que el mal control del hipertiroidismo se asocia a una mayor morbimortalidad neonatal. Es importante anotar que ambos grupos de gestantes recibieron tratamiento con metimazol, siendo regular en el grupo I e irregular en el grupo II. En la literatura existen algunos reportes en los cuales también se demuestra que el tratamiento adecuado del hipertiroidismo durante la gestación con drogas antitiroideas previene el efecto deletéreo del mismo sobre el desarrollo intrauterino $(2,16,17,18)$.

En nuestra serie en relación a la terapia con metimazol no hemos encontrado ningún caso de aplasia cutis neonatal reversible que ha sido descrita en algunas ocasiones $(8,19,20)$. De los 26 neonatos nacidos vivos, dos productos de un embarazo gemelar hicieron bocio que podría atribuirse al metimazol que recibió la madre en dosis de $20 \mathrm{mg} /$ día a partir de fines del segundo trimestre, pero también podrían tener su causa en un exceso de pasaje de inmunoglobulina estimulante de la tiroides de la madre de los fetos ya que la madre no compensó su hipertiroidismo con el tratamiento, recibió una dosis de moderada a baja de metimazol y la exposición fetal al fármaco fue corta por lo tarde del inicio de la terapia en la gestación y la prematuridad de los mismos. El único caso de hipotiroidismo neonatal transitorio de nuestra serie podría estar en relación con el tratamiento con metimazol (20 $\mathrm{mg}$ /día) que recibió la madre en el último trimestre, pero también puede explicarse por el yoduro (lugol: 20 gotas/día) que se le dió a la madre en el periparto, el neonato no tuvo bocio. No hemos encontrado ninguna evidencia clara de que la exposición intra-útero al metamizol a dosis adecuada tenga efectos deletéreos sobre el desarrollo fetal en todo sentido y esto coincide con lo reportado en la literatura $(15,16,2,18,21)$. Es más las tionamidas (propiltiourácilo, metimazol o carbimazol) deben prescribirse en las hipertiroideas gestantes, la dosis debe ser la mínima necesaria para llevarlas al eutiroidismo con niveles de hormonas tiroideas maternas con el rango normal alto. El propiltiourácilo es la droga de elección, por su ventaja adicional sobre las otras tionamidas, pues cruza en menor grado la barrera placentaria, ya que se liga más fuertemente a las proteínas en la sangre materna e inhibe la conversión periférica de $\mathrm{T}_{4}$ a $\mathrm{T}_{3}(19)$.

En lo referente a la terapia con propanolol en nuestra serie no encontramos efecto deletéreo significativo en la gestación y el neonato, sin embargo el escaso número de observaciones y el corto tiempo de exposición, no nos permite hacer conclusiones. En la literatura se han reportado efectos deletéreos por el uso de propanolol, tales como: retardo de crecimiento intrauterino, placenta pequeña, bradicardia fetal, hipoglucemia y deterioro de las respuestas al stress hipóxico $(21,22,23,15)$.

En nuestro estudio encontramos dos neonatos con malformaciones congénitas, ambos productos de madres hipertiroideas descompensadas pertenecientes al grupo II, lo que hace una prevalencia de $10.5 \%$ en este grupo. La causa de las mismas es atribuible al 
hipertiroidismo materno, en el primer caso la madre estuvo hipertiroidea durante toda la gestación y sin tratamiento en el período organogenético crítico; en el segundo, la madre recibió metimazol en forma irregular y permaneció también hipertiroidea durante toda la gestación. En el grupo I (gestantes con enfermedad de Graves compensada con metamizol) no encontramos ningún caso de malformación congénita. Nuestros resultados coinciden con los reportados por Momotani quien encuentra una prevalencia de malformaciones congénitas de $6 \%$ en el grupo de gestantes hipertiroideas y de $0 \%$ en el grupo de gestantes con enfermedad de Graves eutiroideas por tratamiento con metimazol (18).

En conclusión, el hipertiroidismo mal controlado durante la gestación cursa con una mayor morbimortalidad materno-fetal-neonatal; el 75\% de los prematuros, todos los abortos óbitos fetales y las malformaciones congénitas que fueron predominantemente de tipo neurológico se presentaron en el grupo de hipertiroideas mal controladas o no tratadas (grupo II); en el grupo de gestantes compensadas (grupo II); en el grupo de gestantes compensadas o no tratadas (grupo II); en el grupo de gestantes compensadas, del hipertiroidismo, no ocurrieron malformaciones congénitas. El rol benéfico del tratamiento con metimazol sobrepasa el riesgo teratogénico si hay alguno.

\section{REFERENCIAS BIBLIOGRAFICAS}

1. Pineda VG, Rosselot BS, Aguayo J, Cienfuegos G. Hipertiroidismo y embarazo: Un problema diagnóstico y terapéutico. Rev Med Chile 1988; 116: 136-42.

2. David L, Lucas N, Hankins G, et al. Thyrotoxicosis, complicating pregnancy. Am J Obstet Gynecol 1989; 160: 63-70.

3. Lazarus $\mathrm{JH}$, Othman $\mathrm{S}$, Thyroid disease in relation to pregnancy. Clin Endocrinology 1991; 34: 91-98.

4. Holt WA, Talbert LM, Thomas CG, et al. Hyperthyroidism during pregnancy. Obstet Gynecol 1970; 36: 778-85.

5. Becks G, Burrow G, Thyroid disease and pregnancy. Medical Clinics of North America 1991; 75: 121-50.

6. Amino N, Tanazawa O, Mori H, et al. Aggravation of Thyrotoxicosis in early pregnancy and after delivery in Graves disease. J Clin Endocrinol Metab 1982; 55: 108-12.

7. Burrow GN. Hyperthyroidism during pregnancy. N Engl J Med 1978; 298: 150-53.

8. Burrow GN. The management of thyrotoxicosis in pregnancy. N Eng J Med 1985; 313: 362-65.

9. Smith S. Bold A. Interpretation of in-vitro thyroid function test during pregnancy. B J Obstet Gynecol 1983; 90: 532-34.

10. Mestman JH. Thyroid disease in pregnancy. Clinics in Perinatology 1985; 12: 651-67.

11. Mestman JH. Physiology and test of thyroid function En: Sciarra JW. Gynecology and obstetrics. Hagertown: Harper \& Row; 1982.p. 34.

12. Guillaume J, Schussler G, Goldman J. Components of the total serum thyroid hormone 
concentrations during pregnancy: High free thyroxime and blunted thyrotropin (TSH) response to TSH-releasing hormone in the first trimestrer. J Clin Endocrino Metab 1985; 60: 678-84.

13. Bouillon R, Naesens M, Van Asschef, et al. Thyroid function in patients with hyperemesis gravidarum. Am J Obstet Gynecol 1982; 143: 922-25.

14. Lao TTH, Chin RKH, Swainathan R, et al. Erytrocyte Zinc in differential diagnosis of hyperthyroidism in pregnancy. Br Med J 1987; 294: 1064-65.

15. Freedberg IM, Hamoolsky MW, Freedberg AS. The thyroid gland in pregnancy. N Eng J Med 1975; 256: 551-55.

16. Check J. Prenatal treatment of thyrotoxicosis to prevent intrauterine growth retardation. Obstet Gynecol 1982; 60: 122-24.

17. Serup J. Maternal propylthio uracil to manage fetal hyperthyroidism. Lancet $1978 ; 2$ : 896-99.

18. Momontanin N, Ito K. Hamada N, et al. Maternal Hyperthyroidism and congenital malformation in the offspring. Clin Endocrinol 1984; 20: 695-700.

19. Cooper DS. Antithyroid drugs. N Engl J Med 1984; 311: 1353-58.

20. Mutjaba Q, Burrow GN. Treatment of Hyperthyroidism in pregnancy with Propylthiouracil and Metimazole. Obstet Gynecol 1975; 46: 282-86.

21. Burrow GN. Thyroid disease in pregnancy En: Burrow GN, Oppenheimer JH, Volpe R. Thyroid function and Disease. Philadelphia:W.B. Saunders Company; 1989.p. 292-323.

22. Habid A, Mc Carthy JS. Effects on the neonate of propanolol administered during pregnancy. J Pediatrics 1977; 808-11.

23. Pruyn Scphelan JP, Buchanan GC. Long-term Propanolol therapy in pregnancy: Maternal and fetal outcome. Am J Obstet Gynecol 1979; 135: 485-89.

\section{Correspondencia:}

Dr. José Solís Villanueva

Av. Dos de Mayo 649, San Isidro. Lima, Perú.

Teléfono: 440-4426, 438-5167. 